\title{
Research Paper \\ Comparing the Effects of Progressive Muscle Relaxation Technique and Aroma- therapy With Rosemary Oil on Preoperative Anxiety in General Surgery Candidates
}

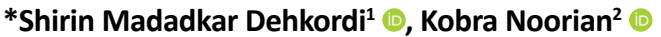

1. Depatment of Nursing, Islamic Azad University, Shahrekord Branch, Shahrekord, Iran.

2. Department of Operating Room, School of Nursing and Midwifery, Shahrekord University of Medical Sciences, Shahrekord, Iran

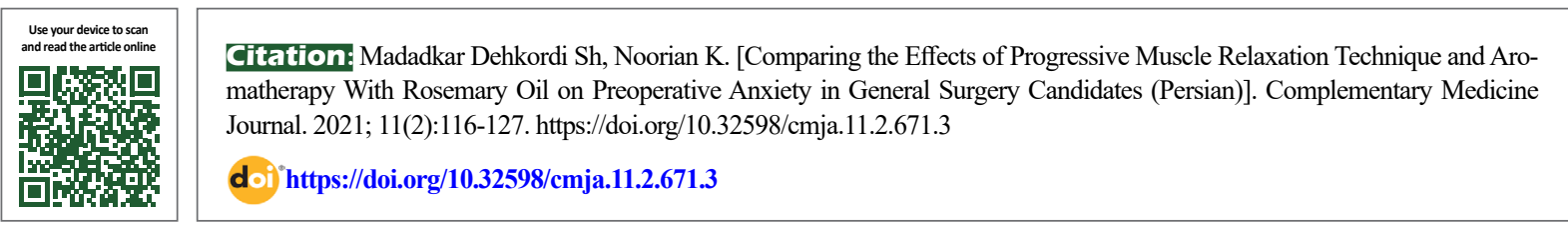

(c) (i) (8)

Article Info:

Received: 03 Jun 2021

Accepted: 10 Jun 202

Available Online: $01 \mathrm{Jul} 2021$

Key words:

Progressive muscle relaxation, Aromatherapy, Rosemary, Anxiety, Surgery

\section{ABSTRACT}

Objective Anxiety is one of the most common problems of patients before surgery. This study aims to compare the effects of Progressive Muscle Relaxation (PMR) technique and aromatherapy with rosemary oil on preoperative anxiety in candidates for general surgery.The aim of this study was to determine the effect of progressive muscle relaxation technique and aromatherapy with rosemary on preoperative anxiety in candidates for general surgery. This study aimed to determine the effect of progressive muscle relaxation techniques and aromatherapy with rose on preoperative anxiety in patients undergoing general surgery was performed. Anxiety is one of the most common problems of patients before surgery. Anxiety is one of the most common disorders in patients prior to surgery

Methods In this clinical trial, participants were 90 patients who were candidates for general surgery referred to Ayatollah Kashani Hospital in Shahrekord, Iran in 2018. After obtaining informed written consent from them, they were randomly divided into three groups: PMR $(n=30)$, aromatherapy $(n=30)$ and control $(n=30)$. The intervention was performed one hour before the surgery. Data collection tools were a demographic form and the Depression, Anxiety and Stress Scale - 21 Items. Collected data were analyzed using chi-square test, paired t-test, and one-way analysis of variance in SPSS v.21 software.In both groups, the intervention was performed by the patient one hour before the operation. In both groups an hour before the operation was performed by the patient. Data collection tools included demographic characteristics questionnaire, 21-item DASS anxiety scale. Data collection tools included demographic questionnaires anxiety scale survey had 21 questions DASS.

In this clinical trial, 90 patients who were candidates for general surgery referred to Ayatollah Kashani Hospital in Shahrekord in 1398 were randomly divided into three groups: relaxation (30), aromatherapy (30) and control (30). In this clinical trial, 90 patients undergoing general surgery hospital Ayatollah Kashani In 1398 randomly into three groups: relaxation (30), aromatherapy (30) and control (30) were divided. In both groups, relaxation and aromatherapy techniques were performed by the patient one hour before the operation. In both groups, relaxation techniques and aromatherapy one hour before surgery was performed by the patient. Data were analyzed using SPSS software version 21. Data using SPSS version 21 was analyzed. Data collection tools included demographic characteristics questionnaire, 21-item DASS Anxiety Scale (DASS 21). Data collection tools included demographic questionnaires anxiety scale survey had 21 questions DASS. In both groups, relaxation and aromatherapy techniques were performed by the patient one hour before the operation. In both groups, relaxation techniques and aromatherapy one hour before surgery was performed by the patient.

Results Mean pre-intervention anxiety score was $16.97 \pm 2.77$ and $16.80 \pm 2.66$ in the PMR and aromatherapy groups, respectively $(P>0.05)$, which decreased to $16.03 \pm 2.54$ and $15.03 \pm 2.88$ after the intervention $(P<0.05)$. There was no statistically significant difference between the two groups in terms of anxiety and the two methods were not superior to each other. But after the intervention, this difference became significant $(P<0.05)$. But after this difference was significant $(P<0.05)$. The results showed that the mean scores of anxiety before the intervention between the three groups were not statistically significant $(P>0.05)$. Results showed that the mean score of anxiety before the intervention showed no significant difference between the three groups $(P<0.05)$. Also, there was no statistically significant difference between the two groups in terms of the studied variable and the two methods of progressive muscle relaxation and aromatherapy were not superior to each other. Also compared between the two groups there was no significant difference variable and progressive muscle relaxation and aromatherapy superior to each other did not.

Conclusion PMR and aromatherapy with rosemary oil can be used in clinics to reduce the level of anxiety in patients before general surgery. According to the results of the study, it can be suggested that relaxation and aromatherapy be used to reduce the level of anxiety of patients before general surgery in the clinic. According to the results, it can be suggested that the relaxation and aromatherapy to reduce the level of public anxiety before surgery was clinical.

\section{* Corresponding Author: \\ Shirin Madadkar Dehkordi}

Address: Depatment of Nursing, Islamic Azad University, Shahrekord Branch, Shahrekord, Iran.

Tel: +98 (913) 9775406

E-mail: shirinmadadkar@gmail.com 


\section{Extended Abstract}

\section{Introduction}

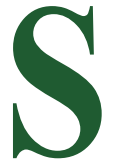

urgery is one of the most widely used treatment methods in many diseases that can be considered as a stressful experience. One of the most common problems of patients before surgery is psychological disorders such as anxiety. Anxiety is seen in a quarter of patients admitted to surgical clinics. The prevalence of preoperative anxiety in adults varies between 11 and $80 \%$. Anxiety as a negative deterrent leads to a decrease in the body's immune system against infections, a tendency to use more painkillers, delays in wound healing and loss of physical and mental energy, a negative impact on the patient's mood, and increased hospital stay.

In general, two types of pharmacological and non-pharmacological methods are used to reduce the level of anxiety in patients. Nowadays, the acceptance of the use of complementary therapies in the health system has increased. Progressive Muscle Relaxation (PMR) technique and aromatherapy are non-pharmacological methods. We found no study on comparing the effects of PMR and aromatherapy with rosemary oil on the anxiety of candidates for general surgery. In this regard, and considering the high prevalence of preoperative anxiety, this study aims to compare the effects of PMR and aromatherapy with rosemary oil on the anxiety of candidates for general surgery.

\section{Materials and Methods}

This research is a clinical trial. The study population consisted of patients who were candidates for general surgery at Ayatollah Kashani Hospital in Shahrekord, Iran in 2018. The sample size was calculated 90 based on previous studies and according to an error rate of 0.05 and test power of 0.80 . The samples were divided into three groups of PMR (30), aromatherapy (30) and control (30) using a random block of 6. Considering the inclusion criteria, the Samples were selected by a convenience sampling method. Data collection tools were a demographic form and the Depression, Anxiety and Stress Scale - 21 Items (DASS-21). In the PMR group, after teaching one hour before the surgery, the patient was asked to listen to the relaxation audio file via a handsfree device. Then, s/he performed the exercises in the presence of the researcher for 20 minutes. In the aromatherapy group, 1 hour before the surgery, 3 drops of $10 \%$ rose essential oil were poured on a paper towel and pinned to the patients' pillows and they were asked to inhale it for 20 minutes. One hour after the intervention and before the surgery, the DASS was completed again in both groups. The control group received no intervention. Data were analyzed in SPSS v. 21 software considering the significance level at 0.05 .

\section{Results}

Chi-square test results did not show a statistically significant difference in demographic characteristics between the study groups $(\mathrm{P}>0.05)$. Paired t-test results showed that PMR and aromatherapy were significantly effective in reducing preoperative anxiety (Table 1). In comparing the mean changes in anxiety scores before and after the intervention between groups, the significance level of one-way ANOVA was reported 0.954 before the intervention and 0.003 after the intervention. Therefore, the score of anxiety after the intervention was different in at least one of the three groups and it can be said that this difference was due to the effect of intervention. According to Duncan's post hoc test results, the mean scores of anxiety after the intervention in the two groups of aromatherapy and PMR were not significantly different, but they were significantly different compared to the control group. Therefore, both interventions had a positive effect on reducing anxiety but are not superior to each other.

\section{Conclusion}

Progressive muscle relaxation and aromatherapy reduce the level of anxiety in patients before general surgery. Considering that, today, the attitude of human societies towards treatment is moving towards traditional medicine and it has

Table 1. Mean anxiety scores before and after intervention in each group

\begin{tabular}{|c|c|c|c|c|c|c|c|c|c|}
\hline \multirow{2}{*}{ Variable } & \multirow{2}{*}{$\begin{array}{l}\text { Assessment } \\
\text { Phase }\end{array}$} & \multicolumn{2}{|c|}{ PMR $(n=30)$} & \multicolumn{3}{|c|}{ Aromatherapy $(n=30)$} & \multicolumn{2}{|c|}{ Control $(n=30)$} & \multirow{2}{*}{$\mathbf{P}$} \\
\hline & & Mean \pm SD & $\begin{array}{l}\text { Std. } \\
\text { error }\end{array}$ & Mean $\pm S$ & & $\begin{array}{l}\text { Std. } \\
\text { error }\end{array}$ & Mean \pm SD & Std. error & \\
\hline \multirow{2}{*}{$\begin{array}{l}\text { Preopera- } \\
\text { tive anxiety }\end{array}$} & $\begin{array}{l}\text { Pre-inter- } \\
\text { vention }\end{array}$ & $16.97 \pm 2.77$ & 0.51 & $16.8 \pm 2.66$ & 2.66 & 0.48 & $84.2 .69 \pm 69$ & 0.46 & $>0.05$ \\
\hline & $\begin{array}{l}\text { Post-inter- } \\
\text { vention }\end{array}$ & $16.03 \pm 2.54$ & 0.46 & $15.03 \pm 2.82$ & 2.82 & 0.51 & $17.40 \pm 2.56$ & 0.51 & $<0.05$ \\
\hline \multicolumn{2}{|c|}{$P$} & \multicolumn{2}{|c|}{$P<0.05$} & \multicolumn{3}{|c|}{$P<0.05$} & \multicolumn{2}{|c|}{$P<0.05$} & \\
\hline
\end{tabular}


always been tried to alleviate patients' anxiety with nonpharmacological interventions, the use of these two methods in clinics is recommended as complementary and inexpensive methods.

\section{Ethical Considerations}

Compliance with ethical guidelines

This clinical trial obtained its ethical approval from ShahrekordUniversity ofMedicalSciences(Code:SKUMS. REC.IR.1396.264) and was registered by the Iranian Registry of Clinical Trials (Code: IRCT20181122041720N1). All ethical principles were observed.

Funding

The study was extracted from a research project approved by Shahrekord University of Medical Sciences.

Authors' contributions

Conceptualization, data collection, editing \& review: Shirin Madadkar Dehkordi; Data analysis: Kobra Noorian.

Conflicts of interest

The authors declare no conflict of interest

Acknowledgements

The authors would like to thank the Vice-Chancellor for Research of Shahrekord University of Medical Sciences, participants, and personnel of surgical wards of Kashani Hospital in Shahrekord for their support and cooperation. 


\title{
مقاله يخووهشى
}

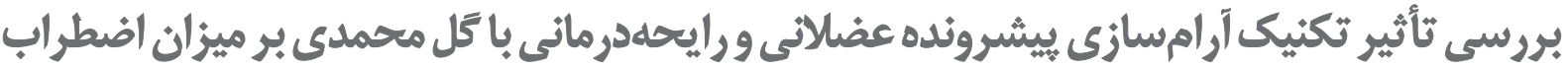

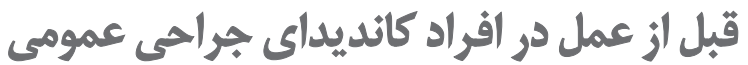

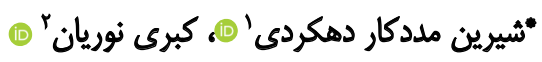 \\ 1. كروه يرستارى، واحد شهركرد، دانشكاه آزاد اسلامى، شهركرد، ايران.

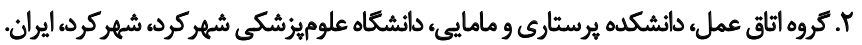

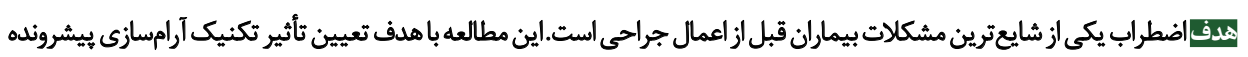

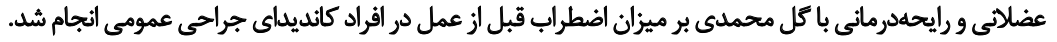

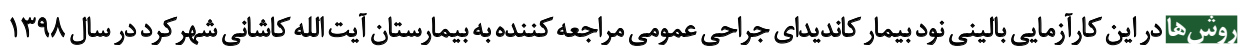

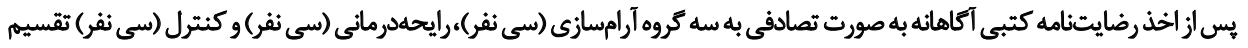

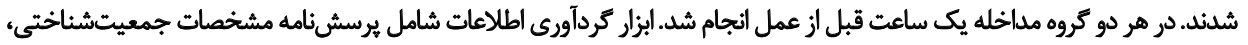

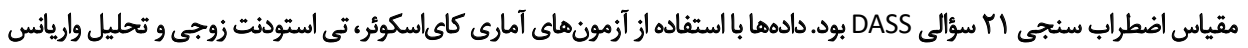

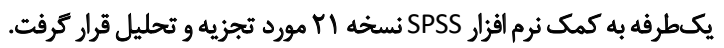

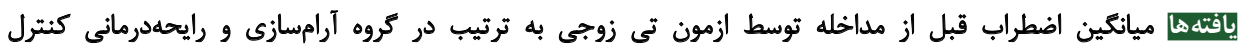

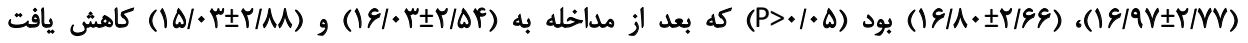

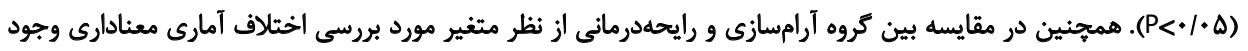

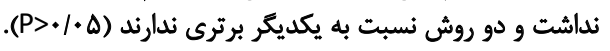

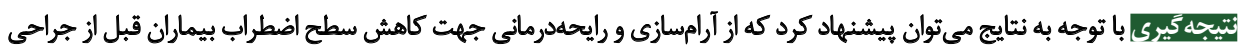
عمومى در بالين استفاده كرد.
\end{abstract}

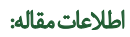

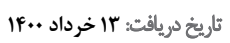

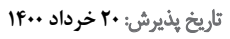

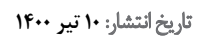

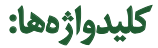

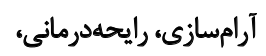
كزل محمدى، اضطراب؛ جراحي
در ايالات متحده آمريكا هر ساله بr ميليون بيمار تحت

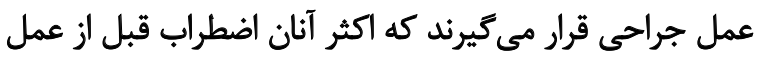

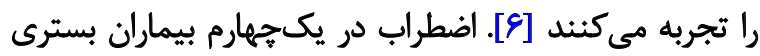

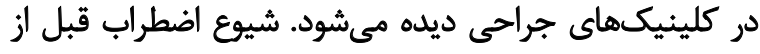

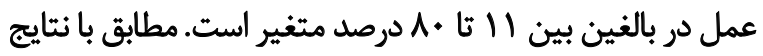

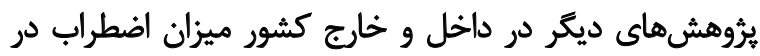

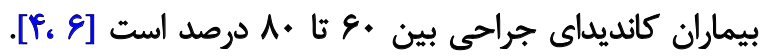

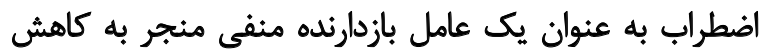

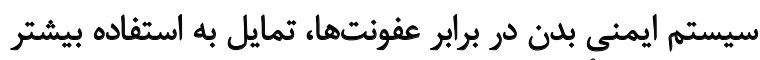

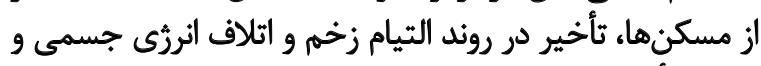

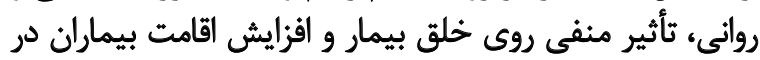

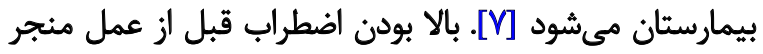

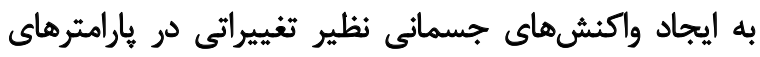

dato

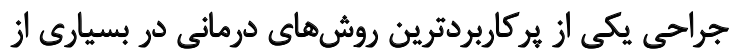

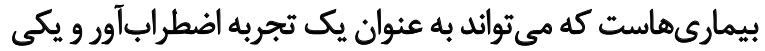

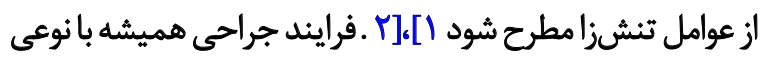

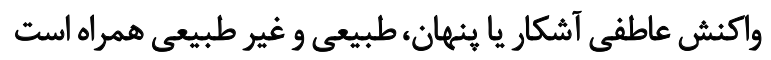

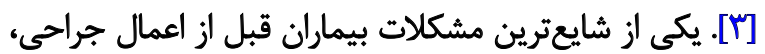

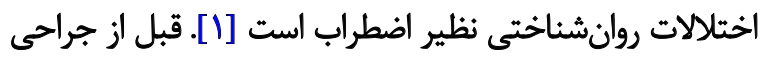

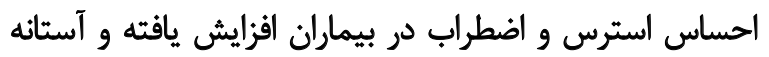

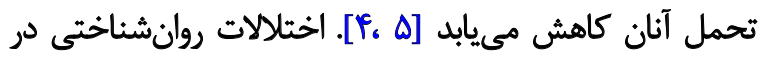

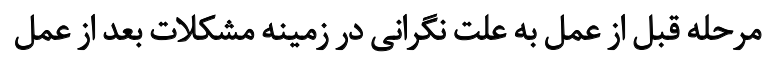

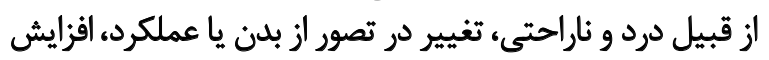

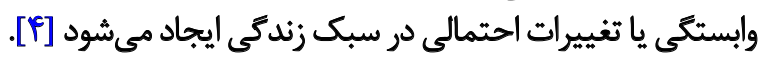




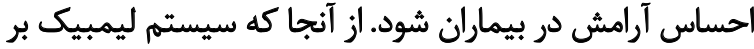

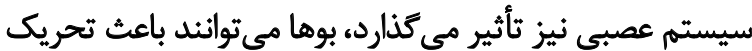

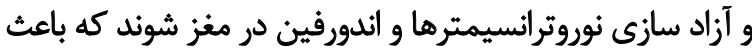

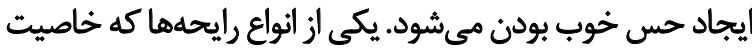

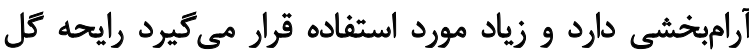

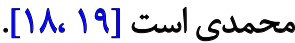

كارداى و همكاران [•r] در يرّوهشى به بروسى تأثيرات

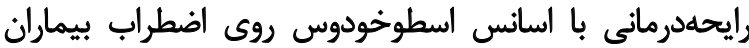

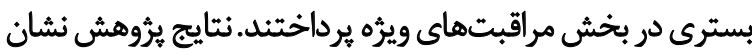

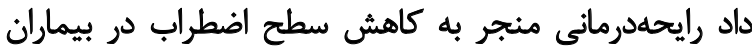

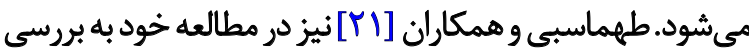

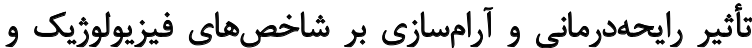

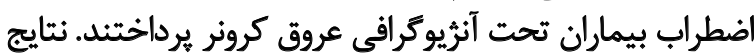

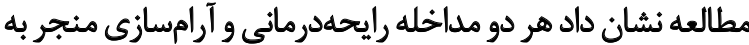

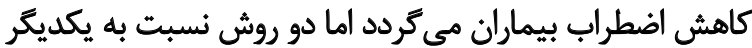

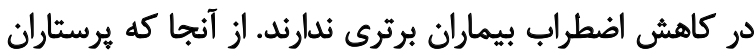

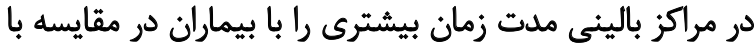

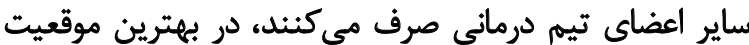

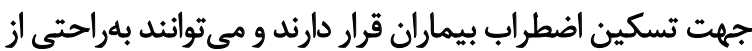

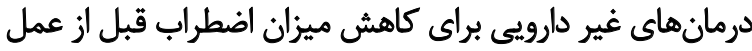

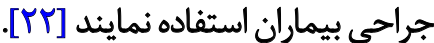

در جستوجوهاى محققين مطالعهاي كه به مقايسه ثأثير

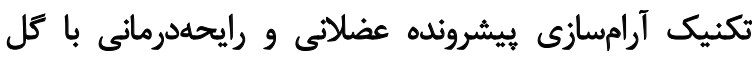

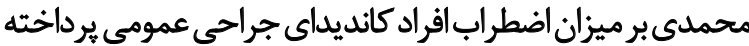

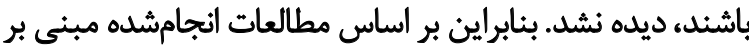

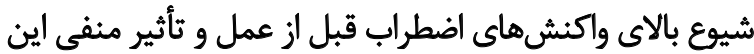

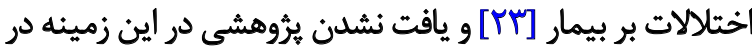

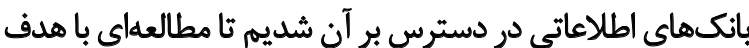

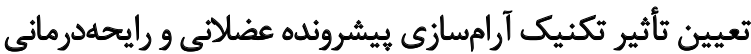

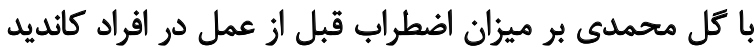

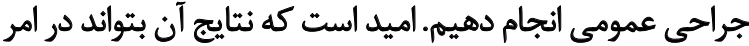
مراقبت از اين بيماران مورد استفاده قرار كيرد.

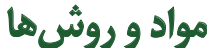

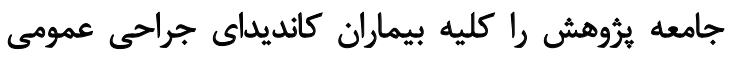

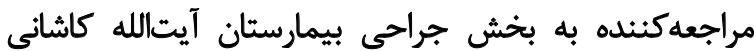

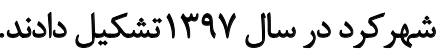

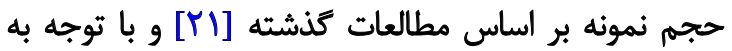

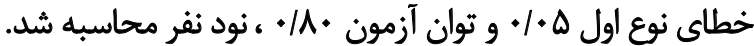

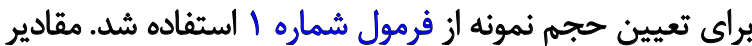
مورد نظر در فرمول جاى كذارى شده است فرول شماره الت
فيزيولوريك بدن مائند تحريك سيسته سمياتيك،افزايش ضربان

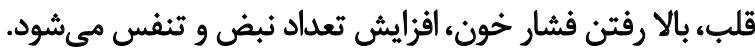

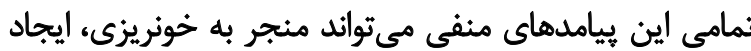

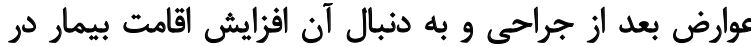

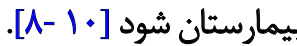

هدف عمده مراقبت در دوره قبل از جراحي، ارتقاى سطح

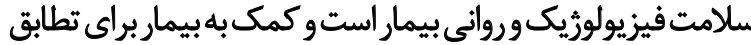

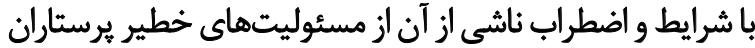

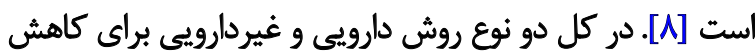

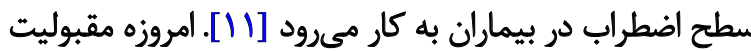

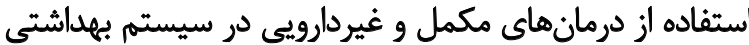

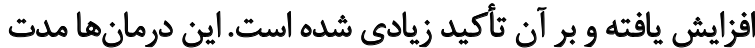

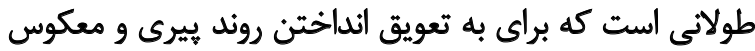

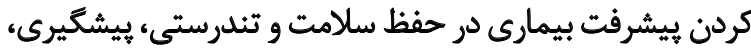

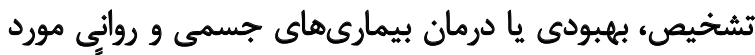

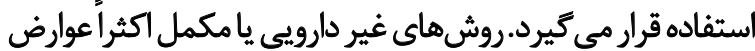

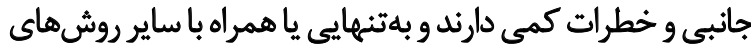

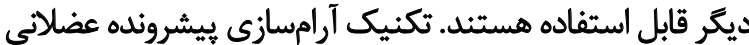

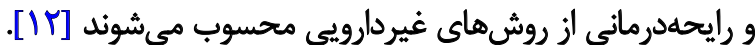

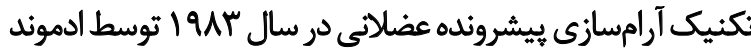

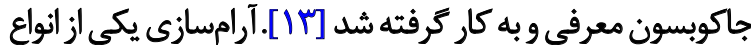

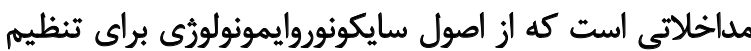

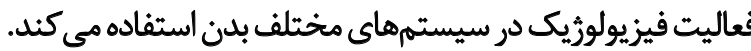

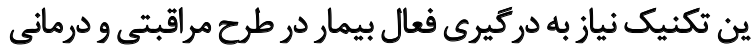

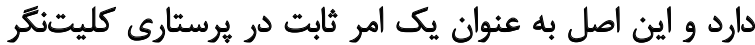

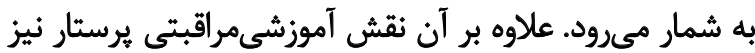

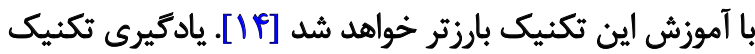

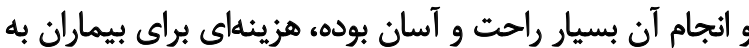

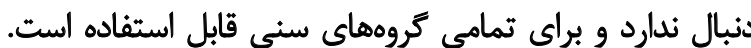

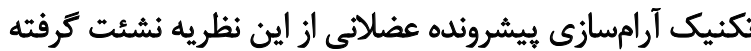

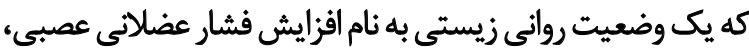

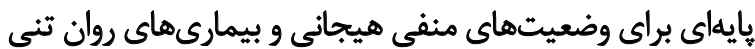

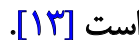

طب رايحهدرمانى نيز يكى از روشهاي غير دارويى و دومين درمان

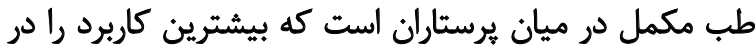

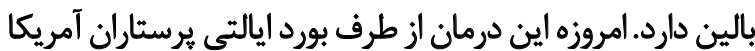

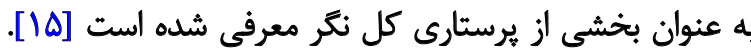

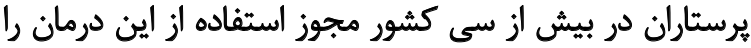

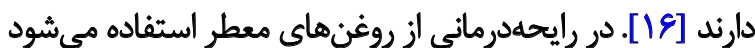

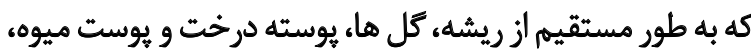

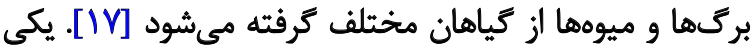

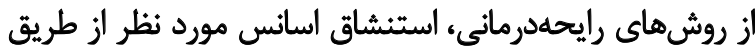

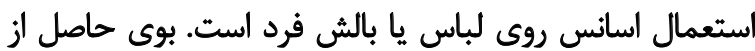

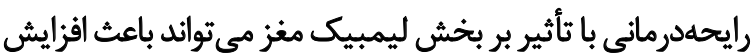


مقياس خودَّزارشى براى ارزيابى حالات عاطفى منفى اضطراب،

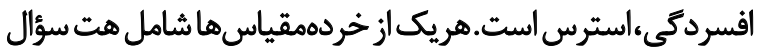

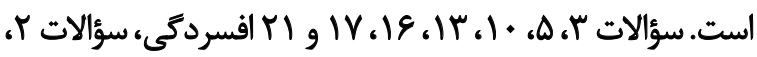

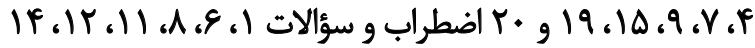

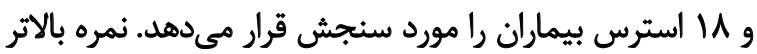

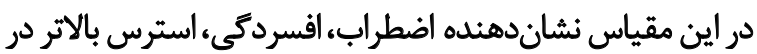

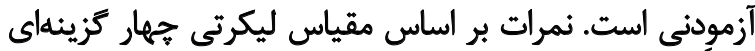

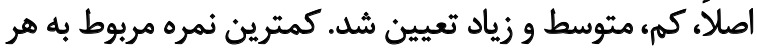

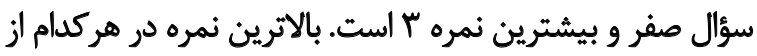

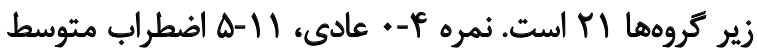

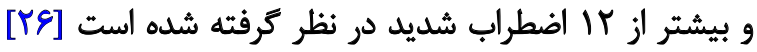

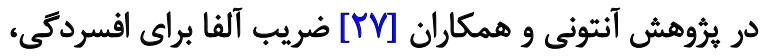

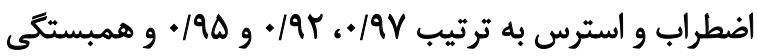

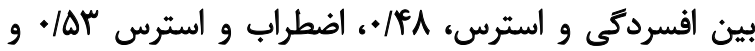

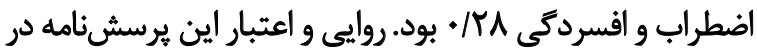

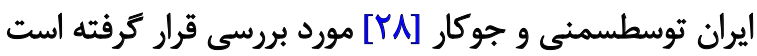

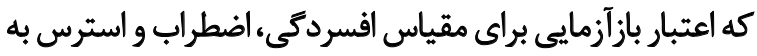

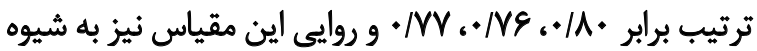
تحليل عاملى از نوع تأييدى مطلوب بوده است وراين.

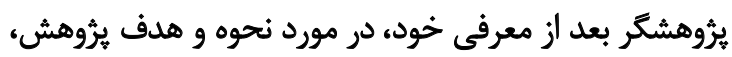

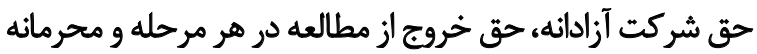

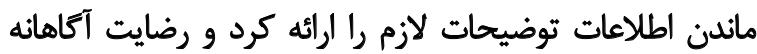

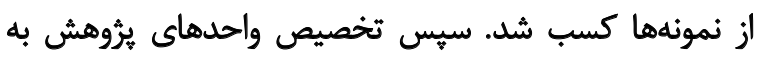

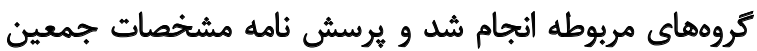

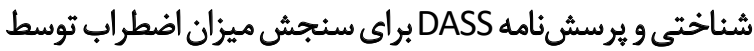

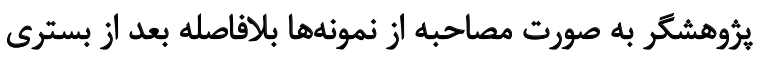

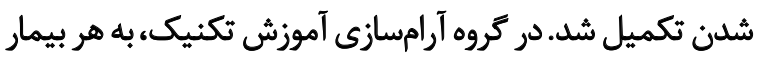

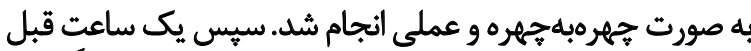

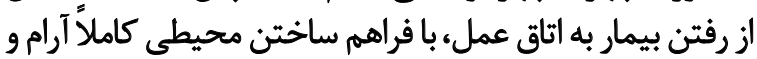

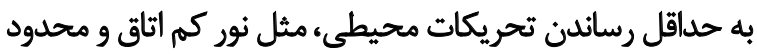

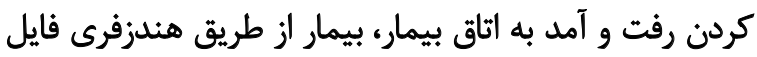

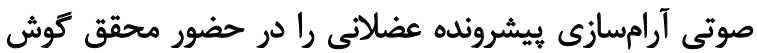

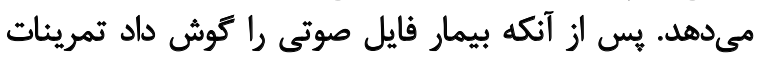

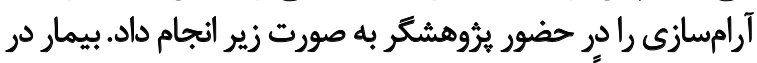

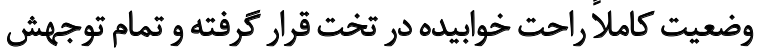

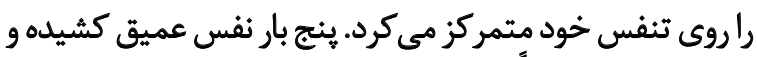

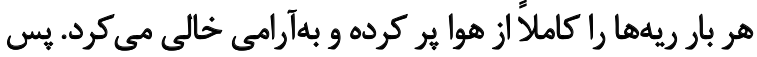

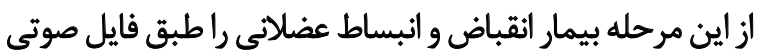

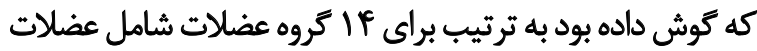

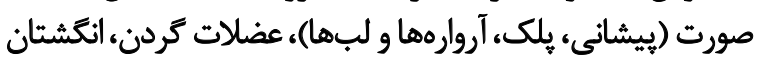

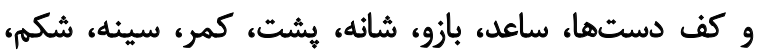

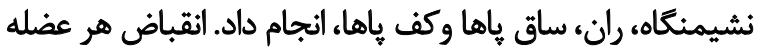

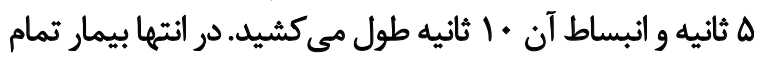

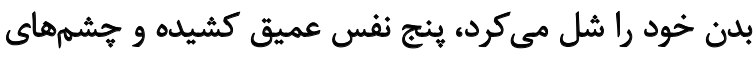

.1

$$
\begin{aligned}
& \mathrm{n}=\frac{\left(\mathrm{z}_{1 \frac{\alpha}{2}}+\mathrm{z}_{1-\beta}\right){ }^{2}\left(\sigma_{1}^{2}+\sigma_{2}^{2}\right)}{2\left(\mu_{1}+\mu_{2}\right)^{2}} \\
& \mathrm{n}=\frac{(1.96+0.84)^{2} \times\left(5.8^{2}+5.65^{2}\right)}{(27.85-23.21)^{2}}=24
\end{aligned}
$$

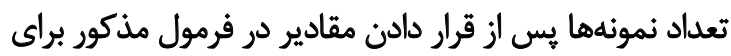

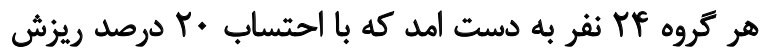

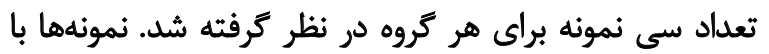

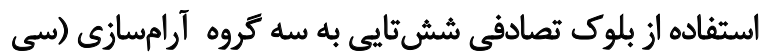

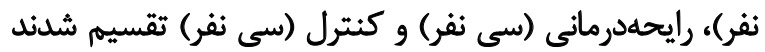

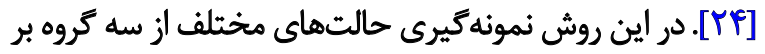

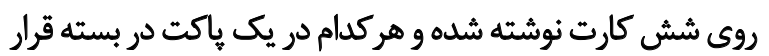

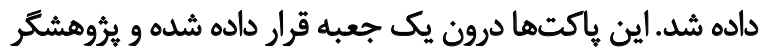

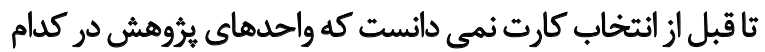

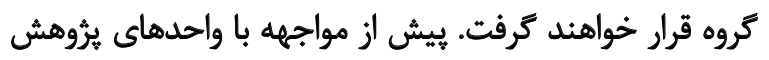

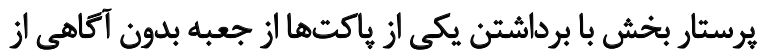

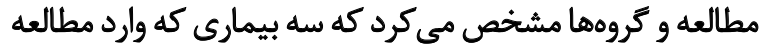
خواهند شد به ترتيب تحت كدام كروه قرار خواهند كرفت كرئ

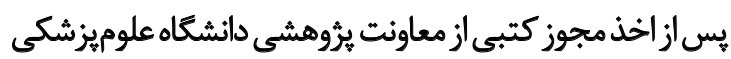

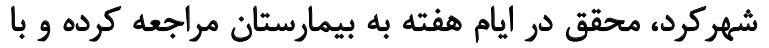

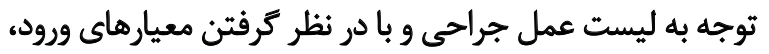

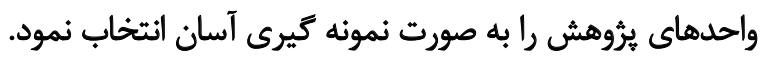

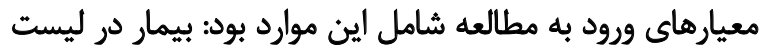

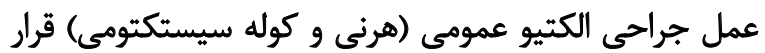

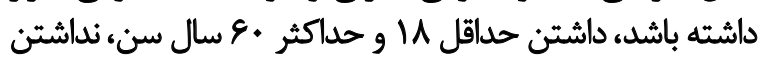

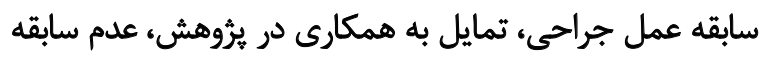

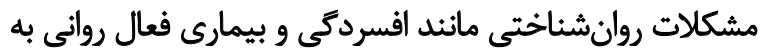

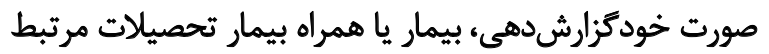

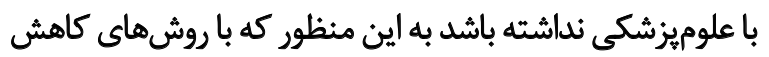

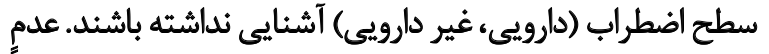

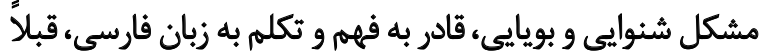

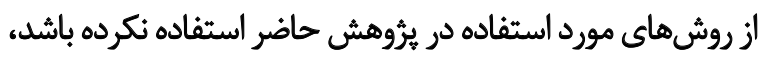

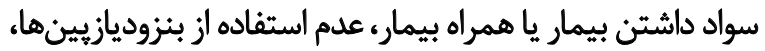

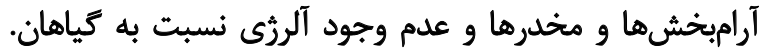

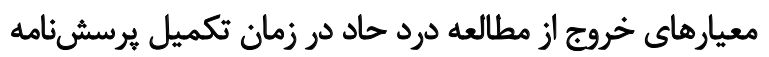
و فوت بيمار بود.

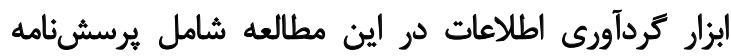

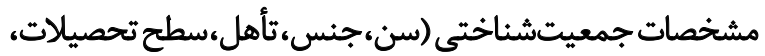

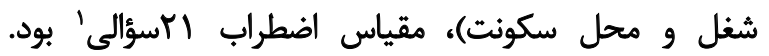

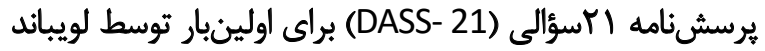

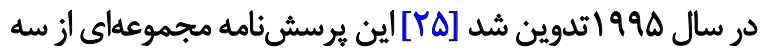

1. Scales21, Depression, Anxiety, Stress-DASS-21 
طبق نتايج بهدست آمده از مطالعه، مشخصات جمعيتشناختى

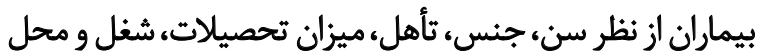

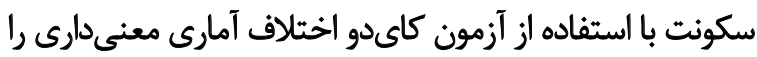

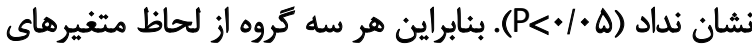
ذكرشده همكن بودند.

در خصوص تعيين و مقايسه ميانكين تغييرات نمرات اضطراب

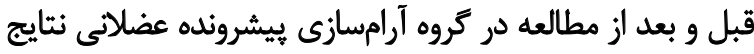

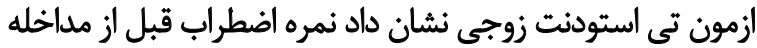

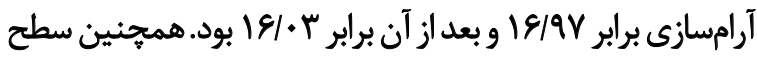

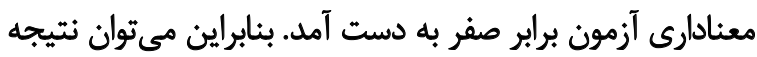

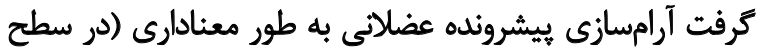

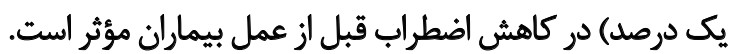
در خصوص تعيين و مقايسه ميانكين تغييرات نمرات اضطراب

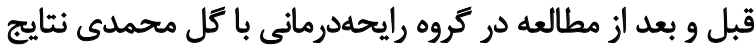

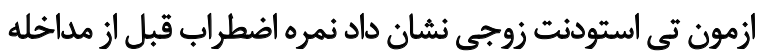

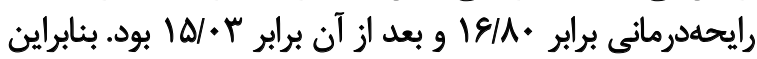

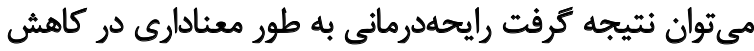

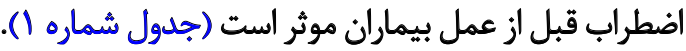
در خصوص تعيين و مقايسه ميانكين تغييرات نمرات اضطراب

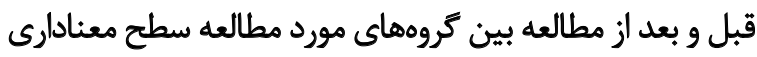

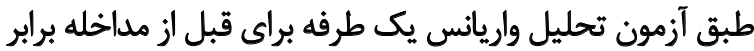

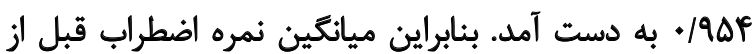

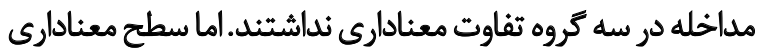

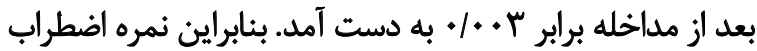

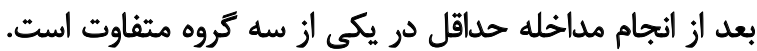

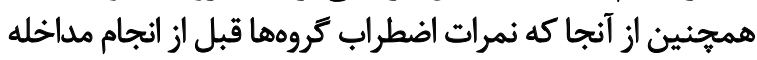

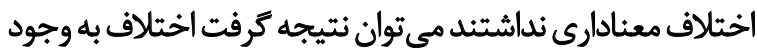

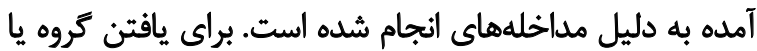
كروههاى متفاوت از آزمون تعقيبي دانكن استفاده شد.
خود را بهآرامى باز مىكرد و به وضعيت عادى برمى بكشت.

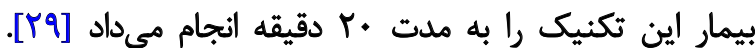

در كروه رايحهدرمانى در روز عمل يك ساعت قبل از رفتن به اتاق

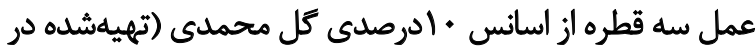

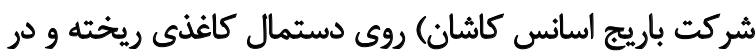

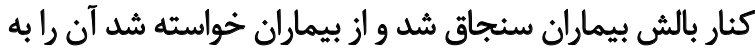

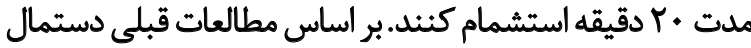

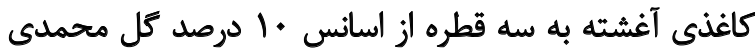

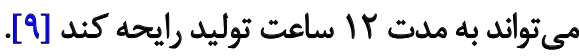

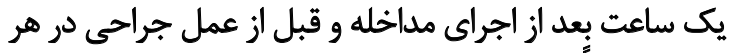

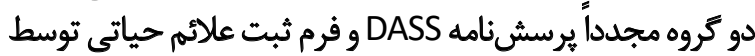

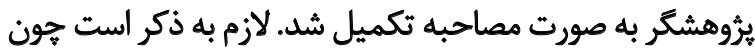

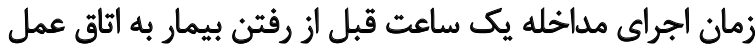

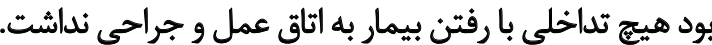
در كروه كنترل مداخلهاى صورت نترفت و تمام مراقبتهاى

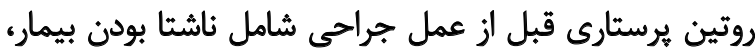

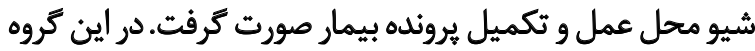

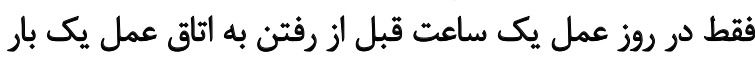

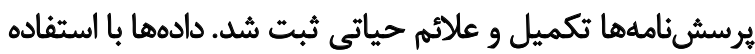

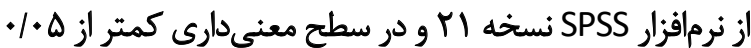

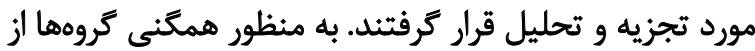

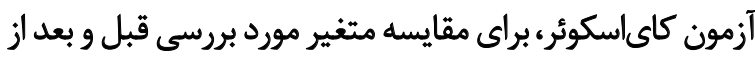

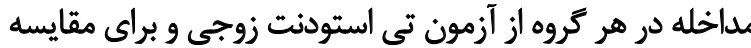

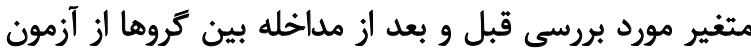
تحليل واريانس يكطرفه استفاده شد (تصوير شماره از ).

يافتهها

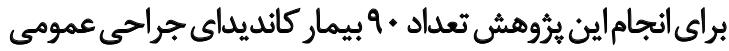

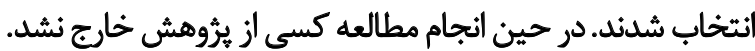


جدول ا. مقايسه ميانكين تغييرات نمرات اضطراب قبل و بعد از مطالعه در هر كدام از تروه آرامسازى بيشرونده عضلانى، رايحلدرمانى و كنترل

\begin{tabular}{|c|c|c|c|c|c|c|c|c|}
\hline \multirow{2}{*}{$\mathbf{P}$} & \multicolumn{2}{|c|}{ كتئرل (سى نفر) } & \multicolumn{2}{|c|}{ رايحهدرمانى (سى نفر) } & \multicolumn{2}{|c|}{ آرامسازى ييشرونده عضلاتى (سى نفر) } & \multirow[b]{2}{*}{ زمان } & \multirow[b]{2}{*}{ 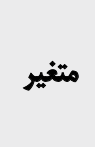 } \\
\hline & استانداى & ميانئيند|انحر اف معيار & اسثاثداري & ميانكين معيار & استاندارئ & ميانئين |نانحراف معيار & & \\
\hline$>.1 .0$ & . pes & VIAFEY/Eq &.$/ P A$ & IE/A土 r/8E &.$|\Delta|$ & $1 \% / 9 V \pm r / W$ & قداخله از & إضط لي \\
\hline$<.1 . \Delta$ &.$(0)$ & $\mid V / P= \pm r / \Delta P$ &.$(0)$ & $10 / \cdot r \pm r / A r$ &.$/ p e$ & $\mid g / \cdot \psi \pm r / \Delta P$ & ملاخله & الصطراب \\
\hline & & $>.1 . \Delta$ & & $<+1 \cdot 0$ & & $<* / \cdot \Delta$ & & \\
\hline
\end{tabular}

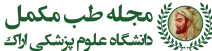

استرس، اضطراب و افسردگى زنان باردار غرداخته بودند نشان

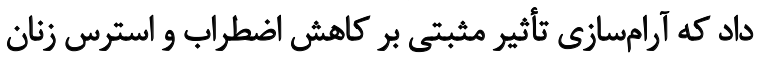

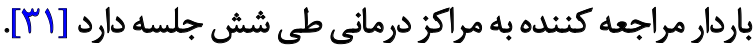
در مطالعه باباشاهى و همكاران [1] بيماران كروه آزمايش دو دور

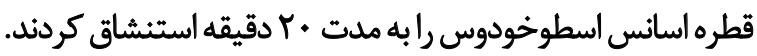

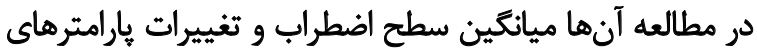

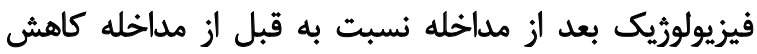

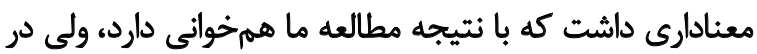

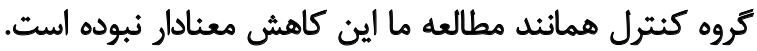
اترجه از نظر نوع روغن مورد استفاده دو مطالعه يكسان نبودماندا. در ساير مطالعات جهت كاهش اضطراب بيمار ان قبل از اعمال

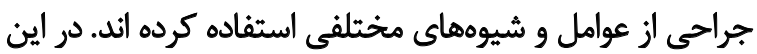

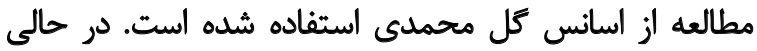

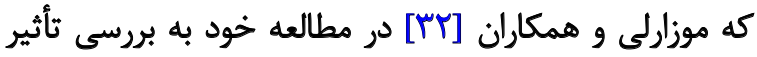

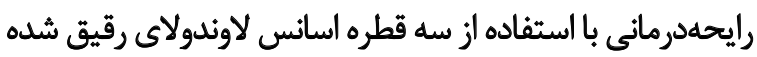

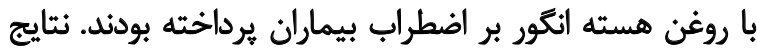

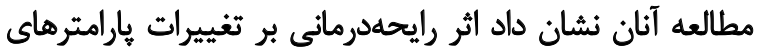

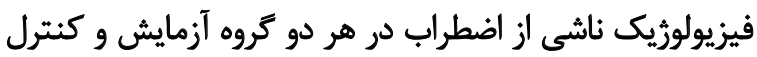

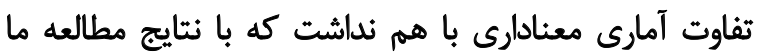

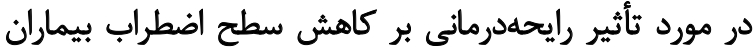

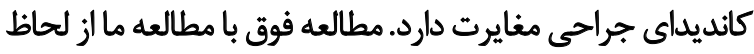
روش كار، نوع رايحه مورد استفاده، مقدار و مدائ استفاده الز إز رايحه تفاوت دارد.

مطالعه سودن و همكاران نيز نشان داد كه رايحهدرمانى ماساريى

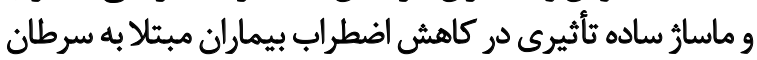

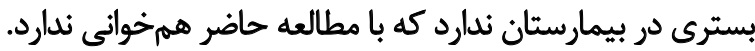

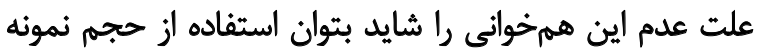

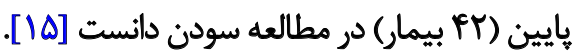

در بررسى مقايسهاى بين كروهى تفاوت آمارى معنى دارى بين

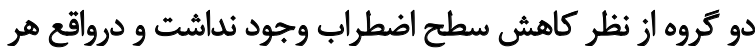

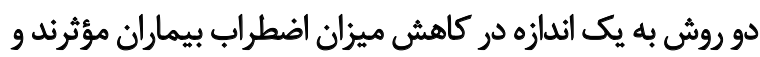
بر ديكرى برترى ندارند.
طبق ازمون تعقيبى دانكن ميانكَين نمرات اضطراب بعد از براز

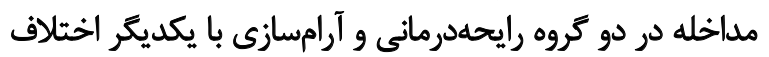

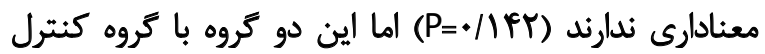

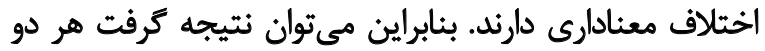

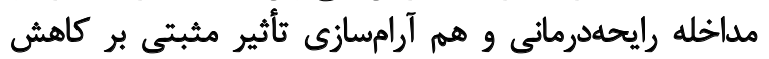

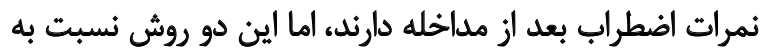

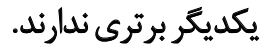

بحث اين مطالعه با هدف تعيين تأثير تكنيك آرامسازى بيشرونده

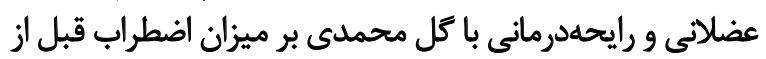

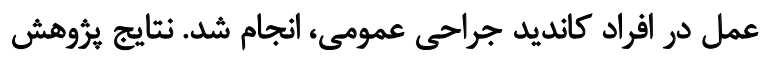

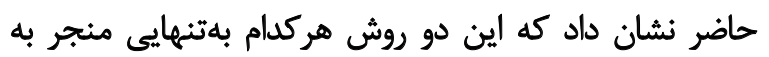

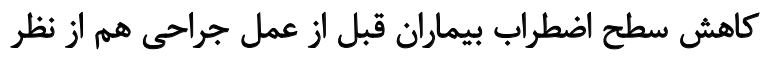

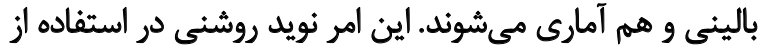

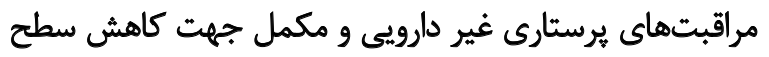

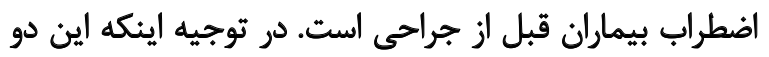

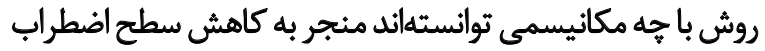

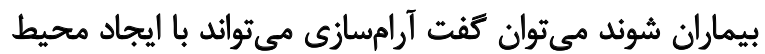

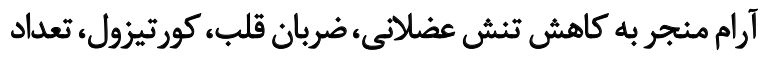

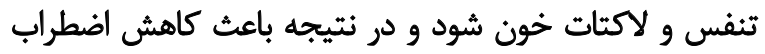

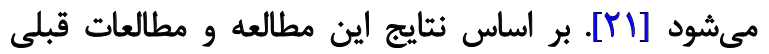

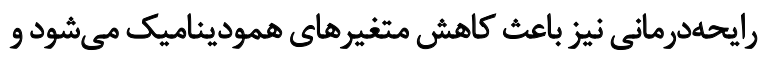

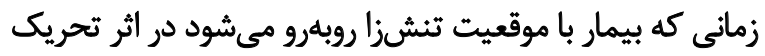

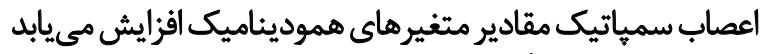

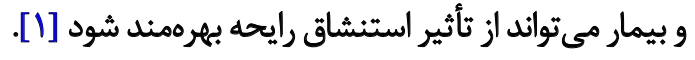

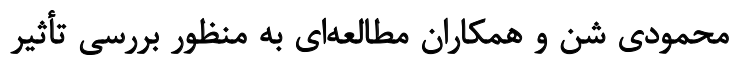

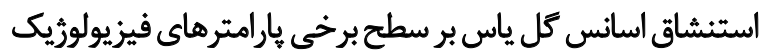

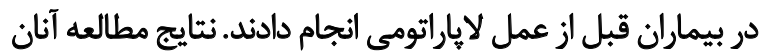

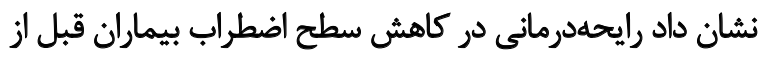

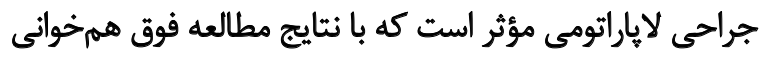

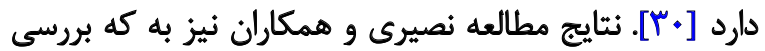

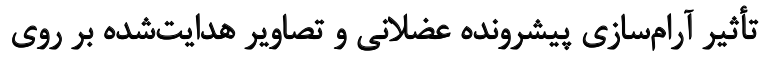


نتايج مطالعه طهماسبى و همكاران با هدف برديى تأثانير

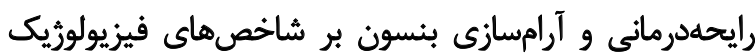
نويسندكان مقاله هيجَّونه تعارضى در منافع اعلام نكردند.

$$
\text { تشكر وقبرداني }
$$

بدين وسيله از معاونت تحقيقات و فناورى دانشعاه علوميزشكى

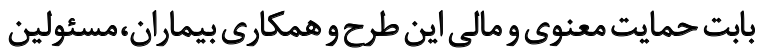

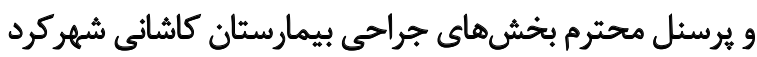
تقدير و تشكر به عمل مى بخث آيد.

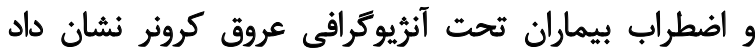

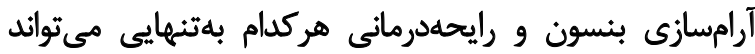

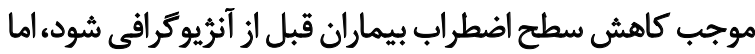

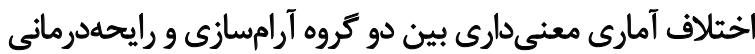

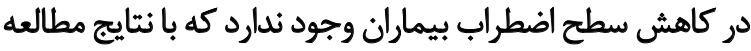
حاضر همخوانى دارد [ [T]

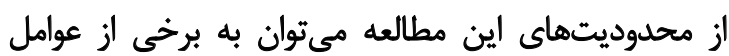

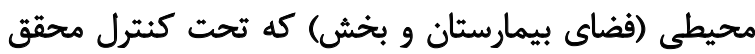

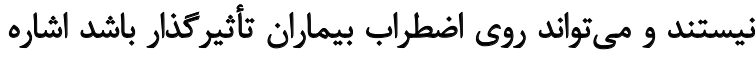

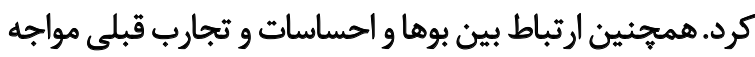

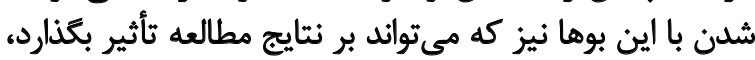

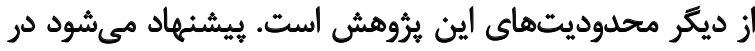

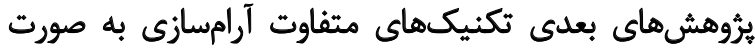

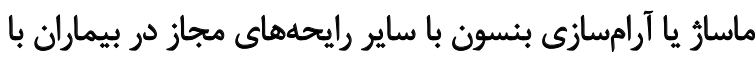
جراحى هاي متفاوت مقايسه شود.

$$
\text { تثيجهنيرى }
$$

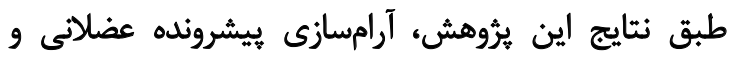

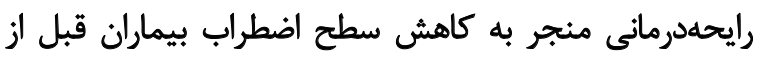

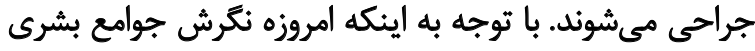

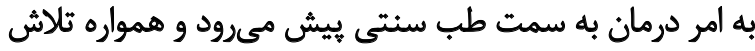

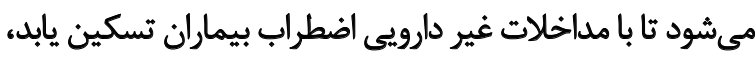

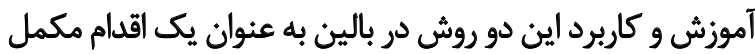

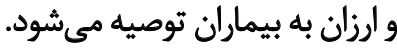

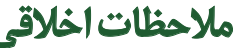

بيروى از اصول اخلاق يُوهشش

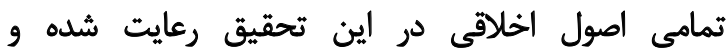

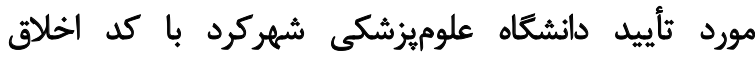

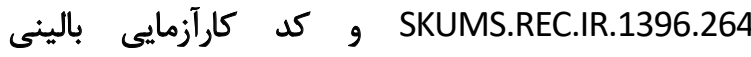
IRCT20181122041720N1

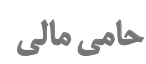

عروهش حاضر بركرفته از طرح يُروهشى مصوب دانشعاه علوميزشكى شهركرد است.

$$
\text { مشاركت ثويسندكّان }
$$

مفهومسازي، جمع آورى دادهها، نكرش و بازبينى متن؛ شيرين مددكار دهكردى؛ تحليل دادمها: كبرى نوريان. 


\section{References}

[1] Babashahi M, kahangi L, Babashahi F, Fayazi S. [Comparing the effect of massage aromatherapy and massage on anxiety level of the patients in the preoperative period: A clinical trial (Persian)]. Evidence Based Care Journal. 2012; 2(2):19-28. https://ebcj.mums.ac.ir/article_395.html

[2] Aghajani M, Mirbagher N. [Comparing the effect of holy Quran recitation and music on patient anxiety and vital signs before abdominal surgeries (Persian)]. Journal of Islamic Life Style. 2016; 1(1):66-87. http:// islamiclifej.com/article-1-105-en.html

[3] Rahimi S, Seidi J, Abdi K, Gholamveisi B. [The relationship between waiting time before surgery and anxiety and vital signs in patients candidate for elective general laparotomy and laparoscopy surgery in hospitals affiliated to Kurdistan University of Medical Sciences in 2019: A short report (Persian)]. Journal of Rafsanjan University of Medical Sciences. 2020; 19(6):633-40. [DOI:10.29252/jrums.19.6.649]

[4] G€ursoy A, Candas B, G€uner S, YIImaz S. Preoperative stress: An operating room nurse intervention assessment. Journal of Perianesthesia Nursing. 2016; 31(6):495-503. [DOI:10.1016/j.jopan.2015.08.011] [PMID]

[5] Zakerimoghadam M, Shaban M, Mehran A, Hashemi S. [Effect of muscle relaxation on anxiety of patients undergo cardiac catheterization (Persian)]. Hayat. 2010; 16(2):64-71. http://hayat.tums.ac.ir/article1-90-fa.html

[6] Rastgarian A, Esmaealpour N, Javadpour S, sadeghi SE, Kalani N, Sepidkar A, et al. [Preoperative anxiety in hospitalized patients: A descriptive cross-sectional study in 2019 (Persian)]. Medical Journal of Mashhad University of Medical Sciences. 2020; 63(1):2209-18. [DOI:10.1016/j. jopan.2015.08.011]

[7] kimiafar Kh, Sarbaz M, Naseri P, Ahmadi Simab S, Abazari F. [The relationship between neurosurgery patients' awareness of surgery procedures and complications on preoperative stress level (Persian)] Journal of Paramedical Science and Rehabilitation. 2017; 5(4):33-42. [DOI:10.1016/j.jopan.2015.08.011]

[8] Torabi M, Salavati M, Pourismail Z, Akbarzade Baghban A. [The effects of acupressure and benson relaxation interventions on pre- operating anxiety in patients undergoing kidney transplantation (Persian)]. Complementary Medicine Journal. 2013; 3(2):441-50. http://cmja.arakmu. ac.ir/article-1-101-en.html

[9] Kamrani F, Nazari M, Sahebalzamani M, Amin G, Farajzadeh M. [Effect of aromatherapy with lemon essential oil on anxiety after orthopedic surgery (Persian)]. Iranian Journal of Rehabilitation Research in Nursing. 2016; 2(4):26-31. [DOI:10.21859/ijrn-02044]

[10] Lin PC. An evaluation of the effectiveness of relaxation therapy for patients receiving joint replacement surgery. Journal of Clinical Nursing. 2012; 21(5-6):601-8. [DOI:10.1111/j.1365-2702.2010.03406.x] [PMID]

[11] Friedberg F. Chronic fatigue syndrome, fibromyalgia, and related illnesses: A clinical modelof assessment and intervention. Journal of Clinical Psychology. 2010; 66(6):641-65. [DOI:10.1002/jclp.20676] [PMID]

[12] Mansour jozan Z, Amini MM. The effect of physical activity and training of progressive muscle relaxation on the level of anxiety and perceived stress in patients with Covid-19 (Persian)]. Sport Psychology Studies. 2020; 9(32):227-48. [DOI:10.1002/jclp.20676]

[13] Conrad A, Roth WT. Muscle relaxation therapy for anxiety disorders: It works but how? Journal of Anxiety Disorders. 2007; 21(3):243-64. [DOI:10.1016/j.janxdis.2006.08.001] [PMID]
[14] Royani Z, Rayyani M, Vatanparast M, Mahdavifar M, Goleij J. [The relationship between self-care and self - efficacy with empowerment in patients undergoing hemodialysis (Persian)]. Military Caring Sciences. 2015; 1(2):116-22. [DOI:10.18869/acadpub.mcs.1.2.116]

[15] Khalili Z, Taraghi Z, Ilali E. [Comparison of the effect of aromatherapy with essential of damask rose and citrus aurantium on the anxaity of the elderly people (Persian)]. Complementary Medicine Journal. 2021; 11(1):20-9. [DOI:10.4103/JNMS.JNMS_39_20]

[16] Jafari-Koulaee A, Ilali E. [The effect of aromatherapy with lavender essence on anxiety control in patients: A systematic review study (Persian)]. Clinical Excellence. 2020; 10(1):21-34. http://ce.mazums ac.ir/article-1-503-fa.html

[17] Ghiasi A, Hasani M, Mollaahmadi L, Hashemzadeh M, Haseli A. [The effect of aromatherapy on labor Pain relief: A systematic review of clinical trials (Persian)]. The Iranian Journal of Obstetrics, Gynecology and Infertility. 2017; 20(2):89-105. [DOI:10.29252/jrums.19.6.649]

[18] Pourmovahed Z, Zare Zardini H, Vahidi AR, Jafari Tadi E. [The effect of inhalation aromatherapy on anxiety level of the patients before Coronary Artery Bypass Graft surgery (CABG) (Persian)] Journal of Rafsanjan University of Medical Sciences and Health Services. 2016; 15(6):551-62. https://www.sid.ir/fa/journal/ViewPaper. aspx?!D=280931

[19] Sánchez-Vidaña DI, Ngai SP, He W, Chow JK, Lau BW, Tsang HW. The effectiveness of aromatherapy for depressive symptoms: A systematic review. Evidence-Based Complementary and Alternative Medicine. 2017; 2017:5869315. [DOI:10.1155/2017/5869315] [PMID] [PMCID]

[20] Karadag E, Samancioglu S, Ozden D, Bakir E. Effects of aromatherapy on sleep quality and anxiety of patients. Nursing in Critical Care. 2017; 22(2):105-12. [DOI:10.1111/nicc.12198] [PMID]

[21] Tahmasebi H, Darvishkhezri H, Abdi H, Abbasi A, Asghari N. [The effect of benson relaxation and aromatherapy on anxiety and physiological indicators in patients unde rgoing coronary angiography (Persian)]. Nursing and Midwifery Journal. 2015; 12(12):1094-103. http://unmf.umsu.ac.ir/article-1-2028-en.htm

[22] Hashemi S, Montazerian M, Nisi L, Bahrampour E. Evaluation of education on patient, $s$ anxiety before abdominal surgical operations. Aligoodarz Nursing Faculty Analytic Research Journal. 2012; 2(2):55 66. http://ndhj.lums.ac.ir/article-1-54-en.html

[23] Zare Marzouni H, Karimi M, Narimi Z, Ghasemi A, Janaki M. [Effects of education on reduction of stress and anxiety of orthopedic surgery (Persian)]. Navid No. 2016; 19(62):62-8. http://nnj.mums.ac.ir/ article_8741.htm

[24] Tahmasbi H, Mahmoodi G, Mokhberi V, Hassani S, Akbarzadeh H, Rahnamai $\mathrm{N}$. The impact of aromatherapy on the anxiety of patients experiencing coronary angiography. Zahedan Journal of Research in Medical Sciences. 2012; 14(3):51-5. https://www.researchgate.net/ profile/Ghahraman-Mahmoudi/publication/278001811 The Impact_of_Aromatherapy_on_the_Anxiety_of_Patients_Experiencing_Coronary_Angiography/links/59cab16945851556-e97e2ec4/ The-Impact-of-Aromatherapy-on-the-Anxiety-of-Patients-Experiencing-Coronary-Angiography.pdf

[25] Lovibond PF, Lovibond SH. The structure of negative emotional states: comparison of the Depression Anxiety Stress Scales (DASS) with the Beck Depression and Anxiety Inventories. Behaviour Research and Therapy. 1995; 33(3):335-43. [DOI:10.1016/00057967(94)00075-U]

[26] Sarafraz M. Shahvand T, Zarea M. [Psychometric properties of Adjustment Disorder New Model-20 (ADNM-20) (Persian)]. Journal of Clinical Psychology. 2018; 9(4):45-54. [DOI:10.1016/00057967(94)00075-U 
[27] Antoni MH, Wimberly SR, Lechner SC, Kazi A, Sifre T, Urcuyo KR, et al. Reduction of cancer-specific thought intrusions and anxiety symptoms with a stress management intervention among women undergoing treatment for breast cancer. The American Journal of Psychiatry. 2006; 163(10):1791-7. [DOI:10.1176/ajp.2006.163.10.1791] [PMID] [PMCID]

[28] Samani S, Jokar B. [Reliability and validity of a short scale of depression, anxiety and stress (Persian)]. Journal of Social Sciences and Humanities of Shiraz University. 2007; 26(3):65-76. https://www.sid.ir/Fa/Journal/ ViewPaper.aspx ?id $=82319$

[29] Jariani M, Saki M, Momeni N. Ebrahimzade F, Seydian A. [The effect of progressive muscle relaxation techniques on anxiety in patients with myocardial infarction (Persian)]. Yafteh. 2011; 13(3):22-30. http://yafte. lums.ac.ir/article-1-559-en.html

[30] Yadegary M, Mahmoodi Shan GR, Vakili MA, Fazel A, Kamkar MZ. [Effect of essential oil jasmine inhalation on physiological index of laparotomy patients in general surgery department (Persian)]. Jorjani Biomed icine Journal. 2017; 5(1):32-41. http://goums.ac.ir/jorjanijournal/ article-1-530-en.html

[31] Nasiri S, Akbari H, Tagharrobi L, Tabatabaee AS. The effect of progressive muscle relaxation and guided imagery on stress, anxiety, and depression of pregnant women referred to health centers. Journal of Education and Health Promotion. 2018; 7:41. [PMCID] [PMID]

[32] Muzzarelli L, Force $M$, Sebold $M$. Aromatherapy and reducing preprocedural anxiety: A controlled prospective study. Gastroenterology Nursing. 2006; 29(6):466-71. [DOI:10.1097/00001610200611000-00005] [PMID] 
This Page Intentionally Left Blank 